\title{
Using Videoconferencing for Elementary/Secondary Teacher Professional Development
}

\author{
Mary Kay Hemenway ${ }^{1}$, Marc Wetzel $^{2}$, Magdalena Rood ${ }^{3}$, Cynthia \\ Roberts-Gray ${ }^{3}$, and Sandra Preston ${ }^{4}$ \\ ${ }^{1}$ Astronomy Department, University of Texas \\ 1 University Station C1400 Austin, TX 78712, USA \\ email: marykay@astro.as.utexas.edu \\ ${ }^{2}$ McDonald Observatory, University of Texas \\ Frank N. Bash Visitors Center, Fort Davis, TX 79734, USA \\ email: Wetzel@astro.as.utexas.edu \\ ${ }^{3}$ Third Coast Research and Development \\ 1108 S. First St Austin, TX 78704 USA \\ email: mrood@thirdcoastresearch.com, CindyRobertsH@aol.com \\ ${ }^{4} \mathrm{McD}$ Donald Observatory, University of Texas \\ 1 University Station C1402 Austin, TX 78712, USA \\ email: sandi@astro.as.utexas.edu
}

\begin{abstract}
Working within a large geographic area, McDonald Observatory lacks the resources to send staff to present professional development workshops to teachers - and only limited numbers of teachers have the resources to attend workshops at our observatory. Our solution is the development of a new program to bring the workshop to teachers in their own communities through videoconferencing. Each workshop location has a co-facilitator who prepared for his/her duties through an orientation/training session held at McDonald Observatory. At the observatory, they experienced a variety of activities and selected the ones most suitable for the grade-levels of the teachers in their region; they recruit the teachers for the local workshop. Each videoconference session includes pre/post assessment of the participants, an interactive videoconference with an expert presenter, and assistance from the co-facilitator who manages the materials and assists with the activities. Through use of this technology, we expect to reach 500 teachers. An independent evaluator is preparing formative and summative evaluation for the project.
\end{abstract}

Keywords. evaluation, professional development, videoconference

\section{Introduction}

Videoconference technology allows us to bring teacher professional development to grade 3-8 teachers across a large geographic (1,244 $\mathrm{km}$ from east to west and 1,289 $\mathrm{km}$ from north to south) region - the state of Texas. These grade levels were chosen because it includes many teachers who are ill prepared to teach science. (Hernandez et al. 2002; Rising 2007). Even well prepared elementary teachers are often lacking in formal education in astronomy, although astronomy appears in the national and state science education standards. The intent of the program is to provide teachers the knowledge and skills to help their students gain confidence and become inspired about science. 


\section{Methodology}

Working with the Texas Regional Collaboratives for Excellence in Science Teaching (TRC), the project had access to 20 Education Service Centers and their infrastructure of 40 institutions of higher education throughout the state. (Barufaldi 2002). Pilot-testing of delivering workshops allowed the education team to determine elements of programming that led to educational gains among the participants and provided feedback concerning participant satisfaction (Preston 2008). It was essential that the videoconference instruction build upon McDonald Observatory's existing educational products and experience. The education team selected activities that required limited amounts of materials to be shipped or purchased and that were related to demonstrated needs in science education standards. The workshops were scheduled for six contact hours (with 45 minutes reserved for evaluation).TRC science specialists were recruited to participate in an orientation session at McDonald Observatory to prepare them to serve as co-facilitators of the videoconference workshops. Their co-facilitating role included recruitment of the classroom teachers for their region, providing some simple materials for videoconference workshop, distribution of materials received from McDonald Observatory for the workshop, trouble shooting during the conference, using knowledge and skills gained through experiencing the activities at the orientation to help train the videoconference participants, and administration of the workshop evaluation instruments. The evaluation included process studies to determine if we reached our target audience, if the program was administered consistent with the plan, and if the resources available met needs of the project; outcome studies in the form of pre- and post-assessments of astronomy knowledge and focus group questionnaires; and summative evaluation integrating results of the process and outcome studies.

\section{Results}

Lessons learned through pilot-testing and mid-course review by the project staff were:

- The videoconference format limits the choice of activities - both due to conditions inherent to videoconference (e.g., lighting) and to cost of supplies to be distributed. Thus, topics such as electromagnetic radiation, optics, and spectroscopy that are very popular at our regular summer residential workshops are best not included.

- The project staff assumed that the science specialists would have sufficient knowledge and experience with videoconference techniques or access to instructional technology staff with those skills. The lack of experience of these science specialists made them uncomfortable in their role (until we arranged for them to view a sample workshop early in the sequence).

- The project staff correctly assumed that some of the science specialists had little experience with teaching astronomy. The initial residential training uncovered some naive ideas, as it was designed to do.

-Participants' comments indicate that activities align with science teaching standards in content and pedagogy.

-The science specialists were generally well prepared to help. Their training experience was motivational. However, those who did not schedule their workshop content during the initial training were reticent about doing so later.

-Science specialists have not been successful in recruiting their full quota of participants (especially in areas impacted by Hurricane Ike). 


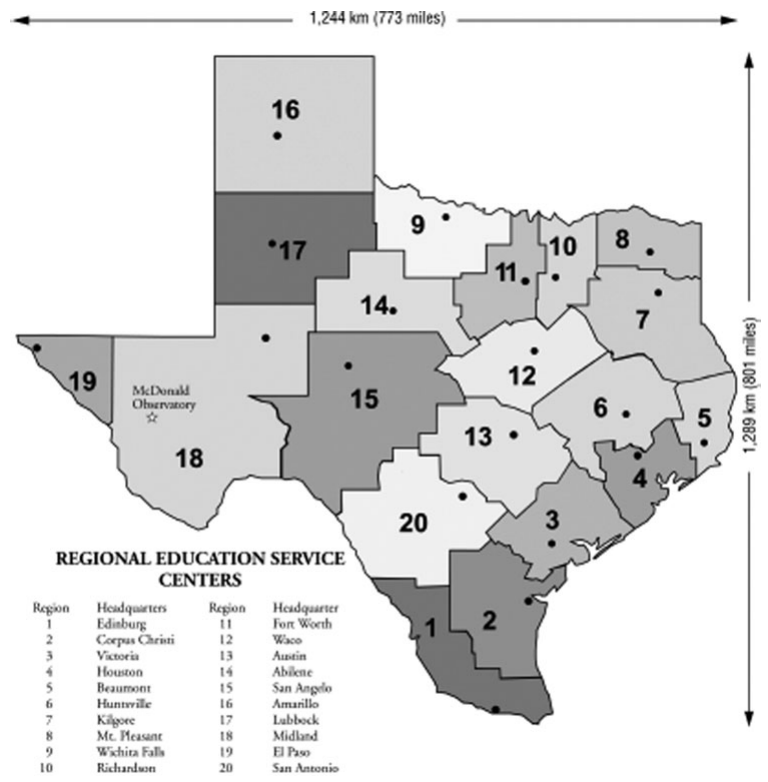

Fourteen potential activities were chosen for the videoconference instructional time. Review of content of the videoconference workshops scheduled for school year 2008-2009 showed the co-facilitators in most regions selected about seven topics/activities. Five workshops were divided between two sessions. Across the 16 workshops presented (two each in Regions 1, 11, and 13; one each in Regions 2, 6, 9, 10, 12, 14, 16, 18 and 20; and a workshop held on-site at McDonald Observatory for Region 18), there were 202 participants ( $88 \%$ female). Of these, $50 \%$ taught grades $\mathrm{K}-5,36 \%$ taught grades $6-8$, and $14 \%$ taught grades 9-12. An additional 15 participants attended portions of workshops but did not participate in the evaluation. A total of 165 of the participants completed both the pre- and post-assessment.

Although not all topics included in the Introductory Astronomy Survey (CAER, 1999; Hufnagel, 2002) were covered in the workshops, it was used as the pre/post instrument. It includes 21 content items and one rating of self-confidence. The mean difference in knowledge from pre- to post- was statistically significant $(t=14.303, p<.01)$. The mean pre- score was 8.89 with scores ranging from 0 to 19 , and the mean post- score was 11.08 with scores ranging from 4 to 20 . Inspection of correct answers on pre- vs. postidentified seven items with substantial increases. All seven address content that was most often included in the videoconference workshops: observing the sun, scale modeling by size and distance, modeling the night sky, gravity, colors of stars, observing the moon, and phases/eclipses. In addition to gaining new knowledge, teachers gained confidence in their test answers. Upon completing the workshop, $43 \%$ were confident or very confident, up from $15 \%$ on the pre-test. Teachers answers to survey items added to the post-test indicated $99 \%$ were highly satisfied or satisfied with delivery of the workshop; $100 \%$ found that all materials and supplies were readily available; $88 \%$ reported the McDonald facilitator went above and beyond expectations in responding to questions; and $89 \%$ indicated the amount of assistance provided matched the groups needs.

Comments from participants were that: "The facilitator took difficult topics and simplified them...made abstract concepts very concrete, which is necessary for the age group we teach" and "This workshop provides hand-on activities, scientific knowledge to increase level of thinking in our students, and the concepts needed to teach our students...we feel 
confident about todays material;" and "My teaching will make more sense to my students because I understand some astronomical concepts more fully;" and "Great workshop! I truly enjoyed it and I can't wait to try these activities with my students;" and "This learning event connects to our Science Objectives in a tremendous way;" and "This was time well spent...the activities are easy to supply and incorporate into our curriculum, ..we like them because they actively engage the learner;" and "Workshop was extremely exciting and informative...presenter challenged group thinking process."

\section{Conclusion}

Videoconference proved useful as a resource for reaching teachers and meeting their professional development needs in astronomy. The workshops were well received. Attrition was relatively low, with most teachers attending the whole workshop, even when it took place on multiple days. Knowledge of astronomy was measurably greater after just the six hours of videoconference workshop activities than it was before. The strategy to train and then task local co-facilitators to plan and organize workshops in configurations that meet local needs appears to have worked well. Further analyses of the focus group discussions and observations/interviews conducted at two of the regional workshops are needed.

We gratefully acknowledge the NASA grant NNX08AG32G, Cynthia and George Mitchell Foundation Education Endowment, the Texas Regional Collaborative for Excellence in Science and Mathematics Teaching, and American Electric Power Foundation for their support of this project.

\section{References}

Barufaldi, J. P. \& Reinhartz, J. 2002, in Models of Science Teacher Preparation. Theory into practice, D. R. Lavoie \& W. M. Roth (eds) (Kluwer: Dordrecht), p. 89

CAER (Collaboration for Astronomy Education Research), 1999, Introductory Astronomy Survey, http://solar.physics.montana.edu/aae/adt/ADTv2.0.PDF

Hernandez, P., Arrington, J., Whitworth, J. 2002, in Proceedings of the Annual International Conference of the Association for the Education of Teachers in Science http://www2.ed. psu.edu/ci/journals/2002aets/02file1.asp

Hufnagel, B. 2002, Astronomy Education Review, 1, 47.

Preston, S., Hemenway, M. K., Wetzel, M., Mace, K. \& Yarbrough, B. 2008, in Communicating Astronomy with the Public, Proceedings from the IAU/National Observatory of Athens/ESA/ESO Conference, L. L. Christensen, M. Zoulias \& I. Robson (eds) (Athens: Eugenides Foundation), p. 500

Whitesides, G. M. et al. (eds) 2007, Rising above the Gathering Storm: Energizing and Employing America for a Brighter Economic Future, (Washington, D. C.: The National Academies Press) 